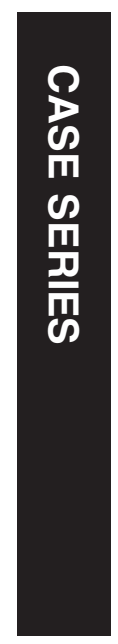

Department of

Ophthalmology, Sheffield

Children's Hospital,

Sheffield, UK

Correspondence:

TKJ Chan, Sheffield

Children's Hospital,

Western Bank, Sheffield

S10 2TH, UK

Tel: +44 (0)114 271 7000;

Fax: +44 (0)114 2717281 .

E-mail: jonathan.chan@sth.

nhs.uk

Received: 28 February 2016 Accepted in revised form:

8 April 2016

Published online:

17 June 2016

\section{Early clinical experience with a new preloaded one-piece intraocular lens in paediatric cataract surgery}

DB Gosling and TKJ Chan

\section{Case reports}

Case 1

A male with left traumatic cataract was operated 10 months after injury, aged 7 years and 8 months. He had anterior capsule fibrosis and irregular pupil, without synechiae. Otherwise fit and well, his preoperative unaided VA was: right 0.160 ; left 0.740 . He underwent uneventful left lensectomy and insertion of +29.50D PCB00 in the bag. The latest post-operative left VA was 0.200 .

\section{Case 2}

A male with bilateral asymmetric developmental lamellar cataract and an X-linked pattern of inheritance who was otherwise well, was referred with left amblyopia for surgery. VA was: right 0.225; left 0.600 (crowded Kay). He was operated when aged 7 years, 6 months. An uneventful left lensectomy and PCB00 +32.00D in the bag were performed. Patching therapy was continued post-operatively with latest postoperative VA 0.350 (crowded logMAR).

\section{Case 3}

Bilateral asymmetric developmental cataract was detected in the male sibling of 'Case 2.' Otherwise fit and well, he presents age 1 year, 8 months with right VA 0.700 and left VA 0.600 (Cardiff cards). Right VA deteriorated to 1.200 over 9 months (left 0.200), despite occlusion therapy. Uneventful right lensectomy and PCB00 +30.50D implant in the bag were performed when aged 2 years, 5 months. Left occlusion therapy was continued and latest post-operative VA was: right 0.700 ; left 0.100 (Cardiff cards). 


\section{Case 4}

A female aged 10 years with bilateral sporadic developmental lamellar cataract, which partially improved with spectacles, was referred from an optometrist. Preoperative BCVA was right 0.575 and left 0.350 (crowded logMAR). She underwent uneventful bilateral sequential lensectomies aged 10 years, 8 months with a +25.50D PCB00 implanted in the bag. Latest unaided post-operative VA was right 0.125 and left 0.100 .

\section{Case 5}

A paediatrician identified bilateral lens opacities, when investigating mild developmental delay and behavioural problems in this 4-year-old male. On assessment, bilateral nuclear and lamellar cataracts were noted with an X-linked inheritance pattern. He had unaided VA of right 0.875 and left 0.825 (crowded Kays). He underwent uneventful bilateral sequential lensectomies and insertion of PCB00 in the bag: right $+29.50 \mathrm{D}$; left $+32.50 \mathrm{D}$, with the aim of achieving monovision. Latest unaided VA was: right 0.400; left 0.500, with spherical equivalent refraction of $+2.00 \mathrm{D}$ right and $+0.50 \mathrm{D}$ left.

\section{Case 6}

Bilateral asymmetric sporadic developmental cataract was seen in a 4 years, 6-month-old male with asthma. His preoperative unaided VA was: right 0.100 ; left 1.800 (crowded Kays). He underwent uneventful left lensectomy and PCB00 +28.50D implanted in the bag. Post-operative unaided left VA was 0.300 .

\section{Case 7}

Right anterior and posterior subcapsular lens opacity was treated in a 14-year-old with left pseudophakia and amblyopia. Preoperative VA was: right 0.200; left 0.650 . Uneventful right phacoemulsification and implant of posterior chamber +29.00D PCB00 were performed when aged 14 years and 9 months. Posterior capsular plaque was noted intraoperatively and Nd:YAG laser posterior capsulotomy was organised for 3 months postoperatively. Latest post-operative right VA was -0.200 .

\section{Discussion}

This is the first case series in the literature using Tecnis PCB00 in children. The AMO Tecnis ZCB00
(Santa Ana, CA, USA) has been shown to have low posterior capsule opacification rates due to its hydrophobic square-edged design, ${ }^{1}$ and its use is established in adult MICS. The Tecnis PCB00 is the same acrylic IOL, but packaged in a preloaded injection device allowing insertion through wounds of $2.2-2.4 \mathrm{~mm}$. The surgeons found the new delivery system helps facilitate a non-touch technique and smaller incisions compared with the ZCB00 and other manually folding IOLs. Using preloaded IOLs may reduce the risk of post-operative endophthalmitis. ${ }^{2}$ Primary posterior capsulectomy is not routinely performed on children who are pseudophakic, although we have an overhead Nd:YAG laser in the operating room, which allows treatment if the posterior capsule opacification is clinically significant. Only one case underwent Nd:YAG laser posterior capsulotomy. This was for capsular plaque identified at the time of initial surgery. This case series raises no concerns about the safety and effectiveness of this device in the context of paediatric cataract surgery.

\section{Summary}

What was known before

- Lensectomy with implantation of an IOL is appropriate in most children with amblyogenic or visually significant cataract over the age of 2 years.

What this study adds

- The AMO Tecnis PCB00 is safe and effective in paediatric cataract surgery.

\section{Conflict of interest}

The authors declare no conflict of interest.

\section{References}

1 Kahraman G, Amon M, Ferdinaro C, Nigl K, Walch M. Intraindividual comparative analysis of capsule opacification after implantation of 2 single-piece hydrophobic acrylic intraocular lenses models: three-year follow-up. J Cataract Refract Surg 2015; 41: 990-996.

2 Weston K, Nicholson R, Bunce C, Yang YF. An 8-year retrospective study of cataract surgery and postoperative endophthalmitis: injectable intraocular lenses may reduce the incidence of postoperative endophthalmitis. Br J Ophthalmol 2015; 99: 1377-1380. 